\title{
Ökobilanz (LCA) - Ein Leitfaden für Ausbildung und Beruf von Walter Klöpffer und Birgit Grahl
}

\author{
Wiley-VCH Verlag GmbH 2009. Praktikerbuch. XIV, 426 Seiten, Hardcover. \\ ISBN-10: 3-527-32043-1. 89,- $€$ (Preis inkl. Mehrwertsteuer zzgl. Versandkosten). \\ http://www.wiley-vch.de/publish/dt/books/bySubjectCH00/ISBN3-527-32043-1
}

\author{
Almut B. Heinrich \\ Online veröffentlicht: 10. Oktober 2009 \\ (C) Springer-Verlag 2009
}

\section{Kurzbeschreibung}

Der Leitfaden zeigt anhand eines konsequent durchgeführten Fallbeispiels (Getränkekarton versus PET-Flasche) das praktische Vorgehen beim Erstellen einer Ökobilanz nach den international gültigen Standards ISO EN 14040 und 14044. Es ist damit Lehrbuch und Praxisleitfaden in einem.

\author{
Aus dem Inhalt \\ Einleitung \\ Was ist eine Ökobilanz? (S 1-2) \\ Historisches \\ Die Struktur der Ökobilanz \\ Normung der Ökobilanztechnik \\ Literatur und Information zur Ökobilanz
}

„Der Begriff Ökobilanz wurde unseres Wissens erstmals 1984 in der Packstoffstudie des damals so benannten Schweizer Bundesamts für Umweltschutz benutzt. Diese Studie hatte einen großen Einfluss auf die weitere Entwicklung der Ökobilanzierung, vor allem im deutschsprachigen Raum (s. Abschnitt 1.2), und daraus resultiert der auch in die Umgangssprache eingedrungene Name für eine Methode, die englisch mit Life Cycle Assessment (LCA) viel besser bezeichnet ist. Die Eindeutschung ,Lebenszyklusanaly$\mathrm{se}^{\text {" hat }}$ sich in den offiziellen Normen nicht durchgesetzt, wird aber gelegentlich gebraucht. Weil das Wort Ökobilanz

A. B. Heinrich $(\bowtie)$

Managing-Editor ScientificJournals, im Auftrag von Springer-Verlag, Kirschgartenstr. 91, 69126 Heidelberg, Deutschland

E-Mail: abh.scientificjournals@googlemail.com vielfach auch für betriebliche Umweltbilanzen benutzt wird, hat man im Zuge der Normung beim DIN (Deutsches Institut für Normung) die genauere Bezeichnung „ProduktÖkobilanz" bzw. ,produktbezogene Ökobilanz“ erwogen, schließlich aber in der mit Österreich und der Schweiz abgestimmten deutschsprachigen Fassung der Norm wieder fallengelassen.

In der Einleitung der internationalen Rahmennorm ISO 14040 wurde die Ökobilanz wie folgt definiert:

Die Ökobilanz ist eine Methode zur Abschätzung der mit einem Produkt verbundenen Umweltaspekte und produktspezifischen potentiellen Umweltwirkungen ... Die Ökobilanz-Studie untersucht die Umweltaspekte und potentiellen Umweltwirkungen im Verlauf des Lebenswegs eines Produktes (d.h. von der Wiege bis zur Bahre) von der Rohstoffgewinnung, über Produktion, Anwendung bis zur Beseitigung.

Ähnlich wie die International Standard Organization (ISO) hatte bereits 1993 die Society of Environmental Toxicology and Chemistry (SETAC) im „Code of Practice“ die Ökobilanz (LCA) definiert.

Ähnliche Definitionen finden sich weiterhin im Grundsatzpapier des DIN-NAGUS und in den Richtlinien, die im Auftrag der skandinavischen Umweltminister erarbeitet wurden, den ,Nordic Guidelines“.

Die bewusste Beschränkung der Ökobilanz auf die Analyse und Auswertung der von den Produktsystemen ausgehenden Umweltwirkungen bringt es mit sich, dass die Methode nur einen, nämlich den ökologischen Pfeiler der Nachhaltigkeit quantifiziert (vgl. Kapitel 6). Die Ausgliederung der ökonomischen und sozialen Faktoren grenzt die Ökobilanz (LCA) von der Produktlinienanalyse (PLA) und ähnlichen Methoden ab. Die Abgrenzung erfolgte, um die Methode nicht zu überfrachten, wohl wissend, dass eine Entscheidung z.B. im Bereich der Entwicklung nachhalti- 
ger Produkte diese anderen Faktoren nicht außer Acht lassen kann und soll.“" (S. 1-2)

Festlegung des Ziels und des Untersuchungsrahmens

\section{Zieldefinition}

\section{Untersuchungsrahmen}

Zielfestlegung im Praxisbeispiel „Vergleich von Getränkekarton und PET-Einwegflasche“"

\section{Sachbilanz}

Grundbegriffe

Energieanalyse

Allokation

Datenerfassung, -herkunft und -qualität

Datenaggregierung und Einheiten

Präsentation der Sachbilanz-Ergebnisse

Sachbilanz im Praxisbeispiel „Vergleich von Getränkekarton und PET-Einwegflasche“

\section{Wirkungsabschätzung}

Grundprinzip der Wirkungsabschätzung

Methode der kritischen Volumina

Die Struktur der Wirkungsabschätzung nach ISO 14040 und 14044

Methode der Wirkungskategorien (Umweltproblemfelder) Wirkungskategorien Wirkungsindikatoren und Charakterisierungsfaktoren

Wirkungsabschätzung im Praxisbeispiel „Vergleich von Getränkekarton und PET-Einwegflasche“

Auswertung, Berichterstattung und kritische Prüfung

Entstehung und Stellenwert der Komponente Auswertung Die Inhalte der Komponente Auswertung nach ISO

Methoden der Ergebnisanalyse

Berichterstattung

Kritische Prüfung

Auswertung im Praxisbeispiel „Vergleich von Getränkekarton und PET-Einwegflasche“"

Von der Ökobilanz zur Nachhaltigkeitsanalyse

Nachhaltigkeit

Die drei Dimensionen der Nachhaltigkeit

Entwicklungstand der Methoden

Ein Life Cycle Assessment oder drei?

Schlussfolgerungen (S. 396-397)

„Es wird oft gesagt, dass schon das Denken in Lebenszyklen ausreicht, um der Umsetzung des Leitbilds Nachhaltige Ent- wicklung näher zu kommen und richtungssichere Entscheidungen nicht immer quantifizierte Informationen erfordern. Dies mag beim Aufzeigen von Brennpunkten stimmen, aber sicher nicht bei der fundierten Entscheidungsfindung weiterhelfen: Wenn verschiedene Lösungsvorschläge gemacht werden, braucht man quantitative Methoden. Es ist die Stärke der Ökobilanz (LCA), dass Quantifizierung möglich ist und dieser Vorteil sollte auch bei der Ergänzung ökonomischer (LCC) ${ }^{1}$ und sozialer Aspekte (SLCA) ${ }^{2}$ erhalten bleiben. Bei der Lebenszykluskostenrechnung wird dies relativ einfach sein, aber schwierig für die produktbezogene Sozialbilanz. Im Hinblick auf die große Bedeutung des Zieles, sollten gleich große Anstrengungen unternommen werden, um die nötigen Werkzeuge zur Verfügung zu stellen und kontinuierlich zu verbessern.

Auch die Ökobilanz selbst ist noch verbesserungsbedürftig und sicherlich verbesserungsfähig (siehe Abschnitt 6.3.1). Dabei sollte, wie in der bisherigen Entwicklung, ein Mittelweg zwischen wissenschaftlich wünschenswerter Genauigkeit und praktischer Machbarkeit eingehalten werden." (S. 396-397)

\section{Über die Autoren}

Walter Klöpffer ist Physikochemiker und einer der Pioniere der Umweltchemie. Er hat schon früh den Begriff der Umwelt im Gegensatz zur Technosphäre klar definiert und das Konzept der Persistenz als einer der ersten definitorisch gefasst.

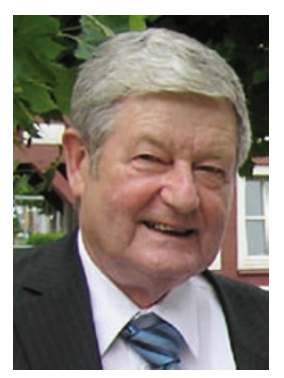

1956-1964 Studium der Chemie an der Karl-FranzensUniversität in Graz, Österreich

1964 Promotion zum Dr. phil am Institut für Physikalische Chemie

1964-1981 Wissenschaftlicher Mitarbeiter am Battelle-Institut e. V., Frankfurt am Main, Abteilung Spektroskopie und Analytische Chemie. Auftragsforschung für Industrie und öffentliche Auftraggeber. Grundlagenforschung im Rahmen des „BattelleInstitute Research in Physical Sciences" und der Deutschen Forschungsgemeinschaft

\footnotetext{
${ }^{1}$ Life Cycle Costing = Lebenszykluskostenrechnung.

${ }^{2}$ Social Life Cycle Assessment $=$ produktbezogene Sozialbilanz.
} 
1975 Habilitation für Physikalische Chemie

1981-1984 Leiter der Zentralstelle Qualität des BattelleInstituts

1983-1985 Leiter der Abteilung Umwelttechnik bzw. Chemie und Ökologie

1986-1992 Leitender Wissenschaftler in der Hauptabteilung Biologie und Umwelt

Seit April 1992 Leiter der Arbeitsgruppe Chemikalien-, Produkt- und Systembewertung der Gesellschaft für Consulting und Analytik im Umweltbereich mbH C.A.U.), Frankfurt am Main

Seit 2003 Freie Konsulententätigkeit (Expert Review nach ISO 14040) in Frankfurt am Main (LCA CONSULT \& REVIEW, Am Dachsberg 56E, 60435 Frankfurt/Main, E-Mail: walter. kloepffer@t-online.de)

Lehrtätigkeit

Lehraufträge an der Universität Mainz in den WS 1971/72 und 1972/73

Seit 1975 nichtbediensteter Professor an der Universität Mainz

Herausgeberschaften

1988 bzw. 1993 Mitglied des „Board of editors“ der Zeitschriften „Umweltwissenschaften und Schadstoff-Forschung, Zeitschrift für Umweltchemie und Ökotoxikologie (UWSF)“ und „Environmental Science and Pollution Research (ESPR)“, beide herausgegeben von Prof. Dr. Otto Hutzinger, Universität Bayreuth, im ecomed Verlag, Landsberg am Lech (heute Springer-Verlag ${ }^{3}$, mit den Herausgebern Prof. Dr. Henner Hollert, Universität Aachen und Prof. Alvin Young, Ph. D., A.L. Young Consultants, Inc., Cheyenne, WY, USA).

1995 Editor-in-Chief der Zeitschrift „The International Journal of Life Cycle Assessment", im ecomed Verlag, Landsberg am Lech, heute Springer-Verlag ${ }^{4}$.

\section{Publikationen}

Klöpffer W (1984) Introduction to polymer spectroscopy, vol. 7, "Polymers, Properties and Applications". Springer, Berlin Heidelberg New York

Klöpffer W (1996) Verhalten und Abbau von Umweltchemikalien - Physikalisch-chemische Grundlagen. Reihe Angewandter Umweltschutz. ecomed Verlag, Landsberg/ Lech

\footnotetext{
${ }^{3}$ UWSF: http://www.springer.com/environment/journal/12302; http:// www.springerlink.com/content/120964

ESPR: http://www.springer.com/environment/journal/11356; http:// www.springerlink.com/content/112851

${ }^{4}$ JLCA: http://www.springer.com/environment/journal/11367; http:// www.springerlink.com/content/112849
}

Klöpffer W, Wagner B (2007) Atmospheric degradation of organic substances. Data for persistence and long-range transport potential. Wiley-VCH

Derzeit ca. 200 Publikationen in wissenschaftlich-technischen Zeitschriften und ca. 240 Vorträge und Vorlesungen.

Birgit Grahl war nach Abschluss ihres Chemiestudiums 1984 an der Universität Hamburg zunächst drei Jahre Bereichsleiterin Chemie beim Öko-Institut e.V. in Freiburg/ Breisgau, dann vier Jahre geschäftsführende Gesellschafterin eines Handelslabors in Hamburg und anschließend freie Konsulentin für Industrie, Behörden und Umweltverbände im Bereich Industrielle Ökologie: Ökobilanz (LCA), Umweltorientierte Produktentwicklung und -kennzeichnung, Umweltmanagementsysteme, Ökocontrolling, Umweltleistungsbewertung, Umweltaspekte nachhaltiger Kommunalentwicklung.

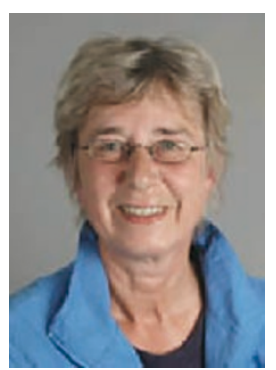

Seit 1997 ist Birgit Grahl Professorin an der Fachhochschule Lübeck im Fachbereich Angewandte Naturwissenschaften.

1999 und 2001 Expertin für Integration und GTZ in der Volksrepublik China zu Themen der ISO14000-Serie

1991-2002 Mitglied in verschiedenen Gremien von DIN, der Deutschen Standard Organisation; maßgeblich beteiligt an der Entwicklung der ISO14000-Serie

Vereidigte Sachverständige für LCA (Handelskammer, Lübeck).

Prüferin im Deutschen System für Akkreditierung und Controllerin der Umweltgutachter im Rahmen von EUEMAS für Deutsche Akkreditierungs- und Zulassungsgesellschaft für Umweltgutachter mbH (DAU GmbH), gemäß $\S 21$ Abs. 1 Nr. 2 Umweltauditgesetz (UAG)

Herausgebertätigkeit

Birgit Grahl gehört dem Editorial Board der Zeitschrift „The International Journal of Life Cycle Assessment" an. Sie ist hier als Gutachterin tätig, trägt eigene Arbeiten bei (s.u., Publikationen) und steht in intensivem Gedankenaustausch mit Walter Klöpffer, wie dieses Buch zeigt.

Die Zusammenarbeit beschreibt Walter Klöpffer wie folgt: „Das Buch war ein echtes Teamwork. Mein Anteil war der Basistext von der Vorlesung her + Interpretation + 
Entwurf zur Nachhaltigkeit und die meiste Literatur. Birgits Anteil waren sämtliche didaktischen Sachen, die Bilder, die Aufgaben und die Aufbereitung des Praxisbeispiels.“

\section{Publikationen}

Schmincke E, Grahl B (2007) The Part of LCA in ISO Type III Environmental Declarations. Int J Life Cycle Assess, Special Issue 12(1):38-45

Schmincke E, Grahl B (2006) Umwelteigenschaften von Produkten. Die Rolle der Ökobilanz in ISO Typ III Umweltdeklarationen. Umweltwiss Schadst Forsch 18(3):185-192

Grahl B, Schmincke E (1996) Evaluation and Decisionmaking Processes in LCA. Int J Life Cycle Assess 1(1): $32-35$

\section{Projekte}

Fitzsimons D., B. Grahl, G. Fleischer (2005). Critical Review according to ISO 14040: Ecological and energetic assessment of re-refining used oils to base oils: substitution of primarily produced based oils including semi-synthetic and synthetic compounds. for GEIR - Groupement Europeen de L'industrie de la Regeneration, Brüssel
Schmincke E., B. Grahl, D. Mutschler (2004). Harmonisierung von Basisdaten für eine ökologische Bewertung von Gebäuden und Bauprodukten. Studie im Auftrag des Bundesamtes für Bauwesen und Raumordnung

Richardsen S., Grahl B. (2001). Anwendernutzen von Umweltkennzahlen als Werkzeug im Umweltmanagement für KMU - Implementierung der kontinuierlichen Verbesserung in übliche Betriebsablaufe. FH Lübeck. Studie, gefördert durch das Umweltministerium Schleswig-Holstein

Grahl B., H. Döhler, K.-H. Feuerherd, H. Wurl (2000). Kritische Prüfung nach ISO 14.040 der Studie „Kunststoffe aus nachwachsenden Rohstoffen: Vergleichende Ökobilanz für Loose-fill-Packmittel aus Stärke bzw. aus Polystyrol“". Im Auftrag der DBU

Grahl B., K. Hedden, U.-J. Möller (2000). Kritische Prüfung nach ISO 14.040 der Studie „Ökologische Bewertung von Altölverwertungswegen“. Im Auftrag des Umweltbundesamtes

Schmincke E., F. Rubik, M. Steinfeld, B. Grahl (2000). Formalisierte und standardisierte Umweltinformationen für Produkte und Dienstleistungen. F+E Vorhaben des Umweltbundesamtes 